\title{
Numerical calculation of the melting phase diagram of low molecular-weight polyethylene
}

\author{
Chinmay Das and Daan Frenkel \\ FOM Institute for Atomic and Molecular Physics, Kruislaan 407, 1098 SJ Amsterdam, The Netherlands
}

(Received 6 January 2003; accepted 26 February 2003)

\begin{abstract}
Using thermodynamic integration, we calculate free energies of the melt and the crystalline phases of a model system of $\mathrm{C}_{198} \mathrm{H}_{398}$ with a realistic all-atom potential. We use the Gibbs-Duhem integration scheme to calculate the melting curve over the experimentally relevant pressure range. The crystal structure and the melting curve obtained from our simulation are in good quantitative agreement with the available experimental results. (C) 2003 American Institute of Physics.
\end{abstract}

[DOI: $10.1063 / 1.1568934]$

\section{INTRODUCTION}

Linear polyethylene (PE) probably is the closest analog of theorists' cherished bead-spring model for polymers. Yet our understanding of even such simple polymers during melting is far from complete. Understanding the melting behavior of polymers is not just of theoretical importance. Properties like rigidity of polymer crystals vary over several orders of magnitude depending on the molecular arrangement of the chains in forming the crystal. A better control of polymer crystallization would have large industrial potential, and understanding the freezing mechanism is a prerequisite for controlling it. Most crystallizable polymers form a lamellar structure in the crystal phase, in which the chains fold back and forth a large number of times. ${ }^{1}$ As to the pathway to the lamellar crystal phase, a number of competing mechanisms have been proposed in the literature. ${ }^{1-5}$ Experiments ${ }^{6,7}$ and simulations of simplified models ${ }^{8,9}$ are not yet in complete agreement on a single mechanism. To understand the freezing mechanism in detail, simulations of realistic polymer models are needed, which can quantitatively reproduce experimentally measured quantities and provide information at the atomic length scale, which is difficult to gather from real experiments. However, even calculations of the equilibrium phase diagram of realistic models of polymers have not been attempted thus far. In this paper we show that currently available model potentials and computational techniques are capable of numerical agreement with real polymer systems in terms of predicting the crystal structure and the melting curve.

By definition, a polymer is a molecule consisting of monomer units in such large numbers that none of the physical properties change significantly with addition or deletion of a few monomer units. The molecules we simulate do not fit into this picture. Real-life polyethylenes normally contain thousands of methylene groups in a single chain and without exception, the chain lengths are polydisperse. Also in long PE molecules, a certain amount of branching is difficult to avoid. However, the most surprising feature of polymer crystallization, that of lamellar crystal formation with temperature dependent lamellar thickness is already observed at the chain lengths simulated in this work. More importantly, ad- vances in synthetic chemistry allow synthesis of essentially monodisperse PE oligomers a few hundred carbon atoms long. We choose a carbon number in this range for the present study because of the existing experimental work on such systems. ${ }^{10,11}$ Certainly, understanding the behavior of monodisperse and strictly linear molecules is easier and we hope that such a model would contain the important features of polydisperse systems, too.

Being a first order transition, accurate calculation of the melting phase diagram involves calculation of the free energy for both the liquid and the crystalline phases. Since only the derivatives of the free energy are measurable quantities, most commonly one performs thermodynamic integration from a system with analytically calculable free energy to the system of interest. ${ }^{12}$ Besides being applied to simple hard particle systems ${ }^{13-16}$ and small molecules, ${ }^{17-19}$ such calculation has been extended to realistic short $n$-alkanes ${ }^{20}$ and model chain molecules. ${ }^{21}$

In the next section, we describe the methodology for free energy calculation and mapping of the phase diagram that we use in our simulations. Section III concentrates on the potential model and the simulation details. In Sec. IV we present the results. We conclude the paper with an outlook on future directions that our work suggests. In the Appendix we reproduce the form of the potential model used in our simulations along with the different parameters of the model.

\section{FREE ENERGY CALCULATION}

\section{A. Solid phase}

To calculate the polymer crystal free energy, we couple each of the atoms to the lattice positions by a set of harmonic springs, with the potential energy given by

$$
\begin{aligned}
\widetilde{U}\left(r^{N}, \lambda\right)= & U\left(r_{0}^{N}\right)+(1-\lambda)\left[U\left(r^{N}\right)-U\left(r_{0}^{N}\right)\right] \\
& +\lambda \sum_{i} \alpha\left(r_{i}-r_{0, i}\right)^{2},
\end{aligned}
$$

such that for $\lambda=0$ the system is the original polymer crystal, while for $\lambda=1$ the system becomes an Einstein crystal with analytically known free energy. In Eq. (1) we have separated 
out $U\left(r_{0}^{N}\right)$, the potential energy of the crystal with all the atoms at their lattice positions ( $N$ is the total number of atoms); $\alpha$ is a spring constant. Because the particles are uniquely identified with their lattice positions, the usual $N$ ! part is missing in the partition function for the Einstein crystal, and the (Helmholtz) free energy is given by

$$
\begin{aligned}
\frac{\beta F_{\text {Ein }}}{N}= & \frac{\beta U\left(r_{0}^{N}\right)}{N}-\frac{3}{2} \ln \left(\frac{\pi}{\alpha \beta}\right)+3 n_{C} \ln \left(\Lambda_{C}\right) \\
& +3 n_{H} \ln \left(\Lambda_{H}\right) .
\end{aligned}
$$

Here, $n_{C}$ and $n_{H}$ are the fractional number of carbon and hydrogen atoms and $\Lambda_{C}$ and $\Lambda_{H}$ are the thermal de Broglie wavelengths for the carbon and hydrogen atoms, respectively. We calculate the chemical potential of the polymer crystal by noting that

$$
\begin{aligned}
\beta \mu(P)=\frac{\beta G}{N}= & \frac{\beta F_{\text {Ein }}}{N}-\frac{\beta}{N} \int_{0}^{1}\left(\frac{\partial F(\lambda)}{\partial \lambda}\right)_{N V T} d \lambda+\frac{\beta}{N} P V \\
= & \frac{\beta F_{\text {Ein }}}{N}-\frac{\beta}{N} \int_{0}^{1}\left(\sum_{i} \alpha\left(r_{i}-r_{0, i}\right)^{2}\right. \\
& \left.-\left[U\left(r^{N}\right)-U\left(r_{0}^{N}\right)\right]\right)_{\lambda} d \lambda+\frac{\beta}{N} P V
\end{aligned}
$$

where $P$ is the pressure and $V$ is the volume of the simulation system. The simulations with the harmonic springs are done with fixed center of mass constraint. In principle, one can add corrections due to fixed center of mass simulations and finite size of the simulation systems. However both the corrections scale as $\ln (N) / N$ with the number, $N$, of atoms and for the present simulation they will be much smaller than the statistical uncertainties in estimating different quantities to calculate the free energy. Hence we do not include them here.

\section{B. Fluid phase}

Most commonly, in calculations of the fluid phase free energy, the original system is expanded isothermally to low enough density such that the individual molecules do not interact with each other. At that limit, one analytically or numerically computes the excess free energy of a single molecule. However, with explicit hydrogen atoms (which leads to branching in the chain), analytical evaluation of even the single-chain free energy is not feasible. In Ref. 20, the authors numerically evaluated the excess free energy of a single (short) chain molecule with respect to an "ideal" chain, which can be evaluated analytically. However, with large chain lengths, we have two hurdles in this route. First, the volume of the melt has to be increased by a substantial amount to reach a density where the chains do not entangle with each other and can be considered as free. Second, the statistical accuracy of brute force calculation of the excess free energy with respect to the ideal chain will run into trouble because there would be many configurations containing overlap of the interaction sites. One can improve this situation by Rosenbluth sampling.
Because of these difficulties, we choose a different reference system for long chain molecules having nontrivial inter- and intramolecular interactions. We slowly switch off all the polymer potentials and at the same time switch on a purely repulsive potential. A purely repulsive potential, with parameters so tuned that when the polymer potential is completely switched off the system goes to a monatomic fluid phase, ensures that during the switching process, the system will not go through another phase transition. Specifically we use a Yukawa potential with the same parameters for all the atoms, and consider the total interaction potential as $\widetilde{U}(\lambda)$ $=(1-\lambda) U^{p m}+\lambda U^{Y}$, where $\lambda$ is a parameter which determines the relative strength of the polymer potential $U^{p m}$ and that of the Yukawa potential $U^{Y}$. The free energy difference between the polymer melt and the Yukawa fluid is calculated by

$$
F^{Y}-F^{p m}=F(\lambda=1)-F(\lambda=0)=\int_{0}^{1}\left\langle\left(U^{Y}-U^{p m}\right)\right\rangle d \lambda .
$$

We choose the parameters for the Yukawa potential such that simple Widom particle insertion can be used to calculate the excess chemical potential, $\mu_{\mathrm{ex}}$. In the switching process the atoms retain their identity to maintain reversibility. However, the polymer potential does not distinguish among the hydrogen atoms bonded to a single carbon atom. Also, either end of the chain can be randomly assigned as the "head" or the "tail" end. So, the Yukawa fluid partition function will have a symmetry factor of $N_{\text {mol }} ! 2^{N_{\text {mer }}-1} 3^{2}$, where $N_{\text {mol }}$ is the number of chain molecules and $N_{\text {mer }}$ is the number of monomer units in a single molecule. The chemical potential of the polymer melt per atom becomes

$$
\begin{aligned}
\beta \mu(P)= & -\ln (V)+\frac{\ln \left(N_{\mathrm{mol}} ! 2^{N_{\mathrm{mer}}-1} 3^{2}\right)}{N}+3 n_{C} \ln \left(\Lambda_{C}\right) \\
& +3 n_{H} \ln \left(\Lambda_{H}\right)+\beta \mu_{\mathrm{ex}}-\frac{\beta}{N} \int_{0}^{1}\left\langle\left(U^{Y}-U^{p m}\right)\right\rangle d \lambda \\
& +\frac{\beta}{N} P V .
\end{aligned}
$$

\section{Phase boundary}

With Eqs. (3) and (5) it is possible to compute the chemical potential $\mu(P)$ at a fixed temperature for both the crystalline and the liquid phase by doing separate simulations at different pressures. The intersection of the two curves determines a single point on the melting phase diagram. Since this calculation of the free energies to locate the phase equilibrium point is rather costly, we use the GibbsDuhem integration scheme ${ }^{22}$ to trace out the phase boundary, i.e., we integrate the Clausius-Clapeyron equation,

$$
\left(\frac{\partial T}{\partial P}\right)_{\operatorname{coex}}=\frac{T \Delta v}{\Delta h},
$$

where $\Delta v=v_{2}-v_{1}$ is the difference in the molar volume between the two phases and $\Delta h=h_{2}-h_{1}$ is the difference in the molar enthalpy. The right-hand side of Eq. (6) can be 
calculated directly from the simulation and the equation can be integrated numerically to map the coexistence line at temperatures different from that at which the free energy calculation is performed.

\section{SIMULATION DETAILS}

Because of the large computational overhead, most computer simulations of realistic polymers use united atom potentials, wherein the monomers are modeled as single pseudoatoms. ${ }^{23}$ This avoids the high frequency modes associated with the stiff interatomic bonds and reduces the number of degrees of freedom. Thus, one can simulate polymers using united atom potentials with longer time steps and less number of computational steps. However, for quantitative calculation of crystalline phase structures, united atom potentials perform poorly. ${ }^{20,24}$

For our simulations, we use the so-called "Flexible Williams" potential ${ }^{25}$ which retains the simplicity of not having many-body interactions and at the same time is shown to perform well for a wide range of molecules both in the fluid and in the crystalline phases. ${ }^{20,25}$ A number of more sophisticated phenomenological and ab initio potential models are available ${ }^{26}$ which give better agreement for certain quantities when compared to the "Flexible Williams" potential. For example, Martonák et $a l^{27}$ find better estimates for the crystal phase density and the lattice parameters than obtained in our simulations. Their potential model uses off diagonal bond-bond, bond-angle, and various angle-angle terms. However, the potential parameters were optimised using a rectangular box and chains that are extended periodically along the $c$-axis. For our calculations, such constrained models are not suitable.

The "Flexible Williams" model uses the nonbonded interactions and the molecular geometries from earlier work by Williams ${ }^{28}$ and the constants for bond stretching and angle bending from work by Smith and Karplus. ${ }^{29}$ A cosine Fourier series fit of the adiabatic torsional potential from ${ }^{29}$ is used as the torsional potential in this model. The detailed form and the parameter values used for the potential are reproduced in the Appendix to this paper. We use a spline fit to make the long ranged nonbonded exp- 6 potential and its derivative go to zero between 5.75 and $6 \AA$ and include long range corrections in the calculation of energy and pressure to compensate for this truncation. In the molecular dynamics simulations we use a multiple time scale scheme ${ }^{30}$ to update the velocities more often from the force due to $\mathrm{CH}$ bond stretching, which has a higher frequency compared to the rest of the interactions. In all the molecular dynamics simulations we monitor the relevant conserved quantity (like energy for microcanonical ensemble) and use a time step such that the fluctuations in the conserved quantity are not greater than one part in $10^{5}$. Typically we use an integration time step of $0.32-0.64$ fs. Within that time step we update the $\mathrm{C}-\mathrm{H}$ bond stretching forces four times in the multiple time scale scheme. The atoms and the barostats are coupled to two separate chains of thermostats, each containing six elements. The thermostat variables are integrated with a higher order algorithm using a time step half the $\mathrm{C}-\mathrm{H}$ bond stretching integration time.
For the calculation of the melt free energy we consider a system of $16 \mathrm{C}_{198} \mathrm{H}_{398}$ molecules in a cubic box with periodic boundary conditions. The polymers were equilibrated at high temperatures $(1000 \mathrm{~K})$ for around $20 \mathrm{~ns}$ using constant isotropic pressure molecular dynamics. ${ }^{31}$ Subsequently the melt was cooled to $435 \mathrm{~K}$ and re-equilibrated for $10 \mathrm{~ns}$ before using for free energy calculations. During this "preparation" stage, we monitor the end to end distance of the chains and the pressure tensor to ensure the system is completely relaxed and all properties are isotropic. The average rootmean-square end-to-end distance $(52.1 \AA)$ and the radius of gyration $(20.2 \AA)$ measured at $435 \mathrm{~K}$ and atmospheric pressure are in agreement with existing literature. ${ }^{32}$ The linear dimension of the average cubic box for the melt simulation at the same conditions is $45.816 \AA$. Since the Cartesian components of the end-to-end distance are smaller than the box length, we expect that the effect of having a small number of chains on the chain conformation and hence, on the melt free energy would be negligible. The melt configuration is equilibrated at the required pressures for $50 \mathrm{ps}$ and in the final box we perform NVT MD simulation to switch off the polymer potential and simultaneously switch on the Yukawa potential of the form $U^{Y}(r)=a_{y} \exp \left(-b_{y} r\right) / r$, with the parameters $\beta a_{y}=3448$ and $b_{y}=5.0 \AA^{-1}$. We performed separate MC simulations of Yukawa particles to make sure that these parameters correspond to the fluid phase far away from the melting line of the pure Yukawa system. To calculate the excess chemical potential of the Yukawa fluid, we make use of the "overlapping distribution method." ${ }^{33,34}$ We perform two separate Monte Carlo simulations: one with $N+1$ particles (system 1) and another with $N$ particles and one ideal gas particle (system 0). For the first system, we calculate the canonical probability distribution, $p_{1}(\Delta U)$, of the energy change for turning a randomly selected particle into an ideal gas particle. For the second system, we calculate the probability distribution, $p_{0}(\Delta U)$, of the energy change for the ideal gas particle to be turned into an interacting particle. Defining $f_{0}(\Delta U)=\ln p_{0}(\Delta U)-\beta \Delta U / 2$, and $f_{1}(\Delta U)$ $=\ln p_{1}(\Delta U)+\beta \Delta U / 2$, the chemical potential $\beta \mu_{\mathrm{ex}}$ is given by $f_{1}(\Delta U)-f_{0}(\Delta U)$. We also compare this estimate with the results of Widom's particle insertion method. ${ }^{35}$ Agreement between the two estimates serves as a test for the density being low enough for the methods to perform accurately.

For the crystal phase simulation, we use 18 molecules in a flexible box with periodic boundary conditions. Initially all the molecules were arranged in an all-trans orthorhombic structure. We use fully flexible NPT MD simulation ${ }^{31}$ to find the actual crystal structure. The initial configuration is equilibrated for $50 \mathrm{ps}$ and subsequent $50 \mathrm{ps}$ is used to calculate the averages at the required pressure. The presence of the $\mathrm{CH}_{3}$ end groups introduce nonequivalence in the lattice positions. So from the NPT simulation we estimate the average box size, and the final configuration is rescaled to have this size. The configuration is equilibrated for 7 ps with NVT simulation and for each atom, the average position is calculated over the next 2 ps. For these NVT simulations, we use unfolded coordinates and keep the center of mass fixed. This average atomic positions are identified with the crystalline lattice for further calculations. For coupling with the Einstein 
TABLE I. Unit cell parameters and specific volume of polyethylene crystal at temperature $293.2 \mathrm{~K}$ and pressure 1 atm. The experimental data are from Ref. 37. The error estimates in the simulation data are in the last digits, as quoted in the brackets.

\begin{tabular}{|c|c|c|c|c|c|c|c|}
\hline & $a / \AA$ & $b / \AA$ & $c / \AA$ & $\alpha$ & $\beta$ & $\gamma$ & $v /\left(\mathrm{cm}^{3} \mathrm{~g}^{-1}\right)$ \\
\hline Expt. & 7.38 & 4.94 & 2.54 & $90^{\circ}$ & $90^{\circ}$ & $90^{\circ}$ & 0.9963 \\
\hline Simul. & $7.4036(5)$ & $4.8443(9)$ & $2.5027(2)$ & $89.43(1)^{\circ}$ & $89.98(1)^{\circ}$ & $89.992(7)^{\circ}$ & $0.9804(2)$ \\
\hline
\end{tabular}

crystal, we fix the center of mass. In our algorithm this simply means that we move all the particles to have the original center of mass and rescale velocities to make the total momentum zero in the position update stage. The spring constant in the Einstein lattice potential is normally chosen so as to make the mean-squared displacement for $\lambda=1$ and $\lambda=0$ equal. ${ }^{12}$ We note that, at $\lambda=0$, since we should be close to the melting line, the Lindemann criterion of having a rootmean-square fluctuation of $10 \%$ of the lattice spacing should hold. This prompts us to fix the spring constant $\beta \alpha$ as 151.7 for $T=435 \mathrm{~K}$. For computing the integral in Eq. (3) we employ a 12 point Gauss-Legendre integration and do simulations at the values of the coupling parameter, $\lambda$, required by the integration scheme.

The use of a small number of molecules is a necessity for simulating large oligomers using an all-atom potential. However, since the number of degrees of freedom is quite large, we believe that the finite size corrections would be small and the results obtained from our simulation will closely correspond to the bulk values.

\section{RESULTS}

One of the essential requirements for a potential model to accurately predict the free energy of the crystalline solid is that the crystal structure measured in simulation should be close to the experimentally measured values. To begin with, we arrange the chains in an orthorhombic structure with lattice parameters close to the experimentally measured values. After an equlibration period of $200 \mathrm{ps}$ with flexible NPT MD simulation, we calculate the lattice parameters over 50 ps and estimate the statistical errors using block analysis. ${ }^{36}$ The $\mathrm{CH}_{3}$ end groups introduce some ambiguity in the calculation of the lattice parameter along the chain direction. Here we report the average distance between the chain ends divided by the number of lattice sites along the $c$ direction as the lattice parameter $c$. In Table I we present the results for simulation at $T=293.2 \mathrm{~K}$ and $P=1 \mathrm{~atm}$. The unit cell parameters in Table I are close to the experimental results. The largest deviation is in the $b$ dimension (we underestimate $b$ by around $2 \%$ ). The crystalline structure also deviates slightly from the orthorhombic symmetry in our simulation. However, the agreement is good enough to use the simple potential for quantitative calculations of polyethylene. As noted in Ref. 25, a better agreement with experimental data is only possible if one includes chain length dependent and possibly anharmonic potentials. In Fig. 1, we show a snapshot of the crystalline phase from the simulation.

To locate the coexistence pressure, we calculate free energies of the melt and the crystal at $435 \mathrm{~K}$, which is about 20 $\mathrm{K}$ higher than the melting temperature for infinite chain $\mathrm{PE}$ at ambient pressure. This step involves a large number of separate simulations at different pressures. Here we show only representative results, from simulations at $400 \mathrm{MPa}$.

In Fig. 2 we plot the integrand in Eq. (3) as a function of $\lambda$ for $P=400 \mathrm{MPa}$. The integrand has a mild peak at $\lambda=0$. The behavior of the integrand depends on the value of the spring constant chosen for the Einstein crystal. As noted in previous calculations for large macromolecules, ${ }^{21}$ finding a spring constant which removes the peaked behavior at the both extreme end ranges of $\lambda$ is not possible. For $P$ $=400 \mathrm{MPa}$, we have used a 10 point integration scheme in addition to the 12 point quadrature used for all the pressures. The results of the two separate calculations match within $0.01 k_{B} T$ per particle. Thus the presence of a mild peak at $\lambda=0$ does not hinder accurate convergence of the quadrature.

For the calculation of the melt free energy (Fig. 3), we take a system of simple Yukawa particles as the reference. This involves slowly switching off the bonded potentials and simultaneously switching on the Yukawa potential. In the limiting case of zero bonded potential $[\lambda=1$ in Eq. (4)], the bonded particles can move arbitrarily far away, leading to very high energy configurations for the polymer bonds. In principle the integrand diverges for harmonic bonds. But because of the periodic boundary conditions, in the folded coordinates the bonded potentials are also periodic. So, the integrand becomes large but remains finite even when the polymer potentials are completely switched off. We divide

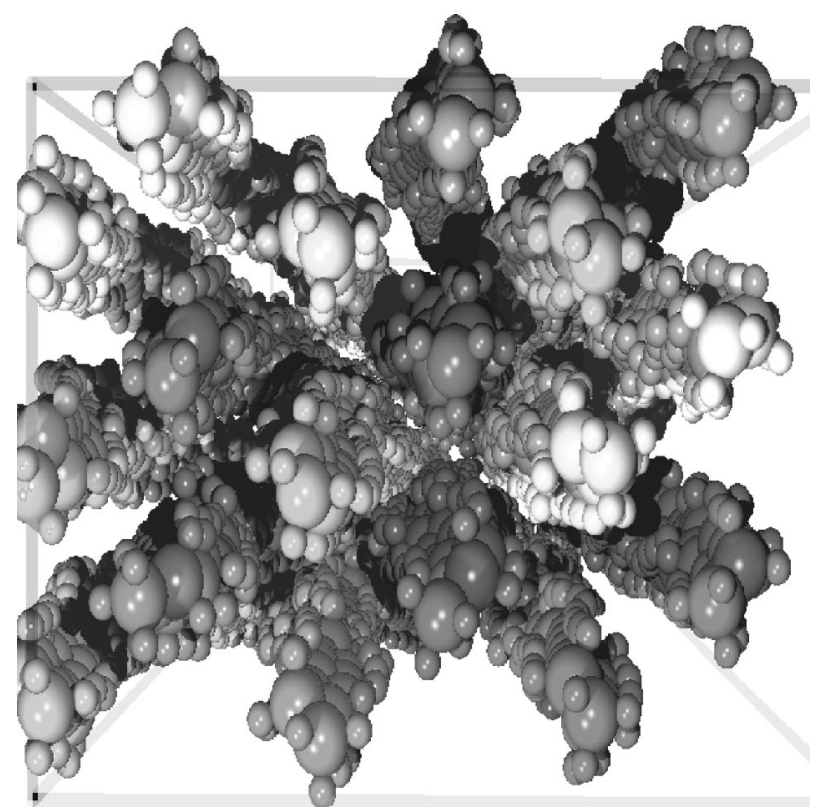

FIG. 1. Simulation snapshot of crystalline PE. The view is from one end along the $c$ axis at atmospheric pressure and temperature of $293.5 \mathrm{~K}$. 


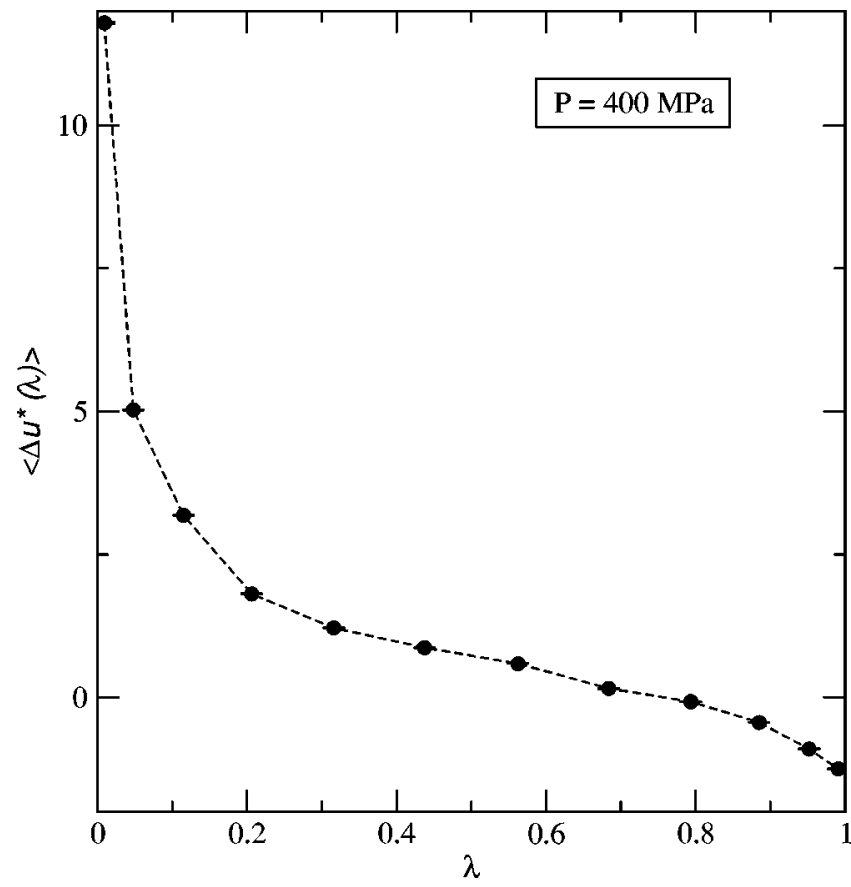

FIG. 2. $\left\langle U^{*}(\lambda)\right\rangle \equiv(\beta / N)\left\langle U_{\mathrm{Ein}}-\left(U-U_{0}\right)\right\rangle_{\lambda}$ as a function of the coupling parameter $\lambda$ for $P=400 \mathrm{MPa}$ and $T=435 \mathrm{~K}$.

the range of the integration into three zones such that the contribution to the integration is roughly equal from each of the zones. In Fig. 4 we plot the integrand as a function of $\lambda$ in the three integration zones separately. Note that in panels

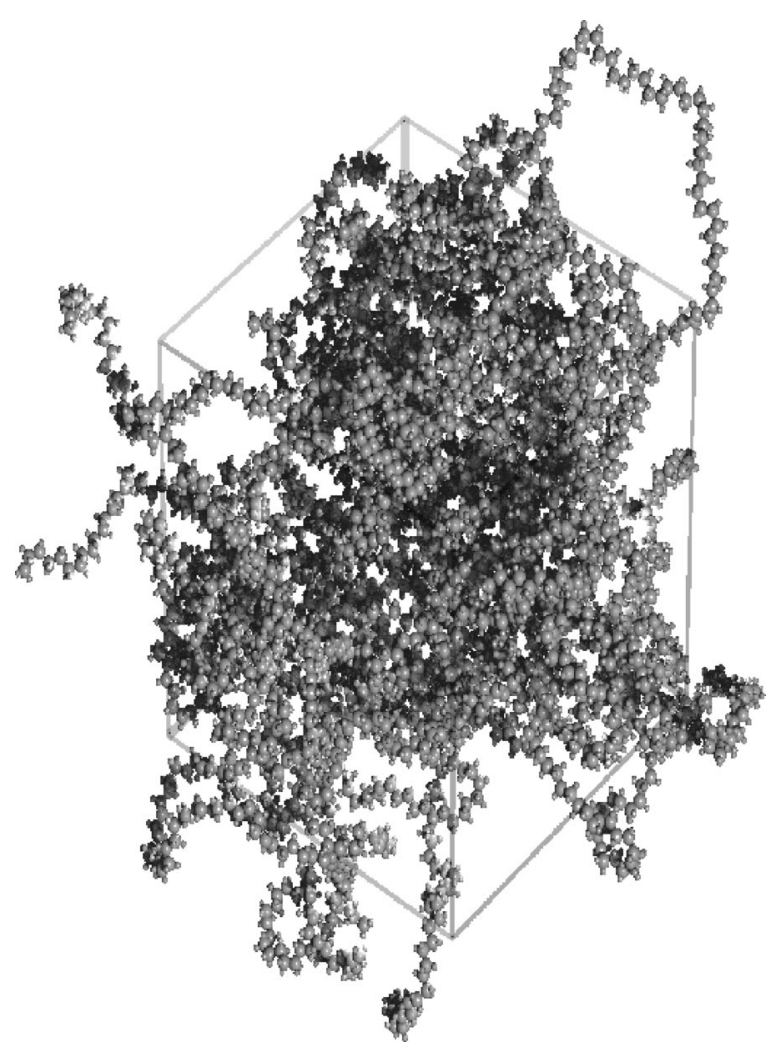

FIG. 3. Simulation snapshot of PE melt at $435 \mathrm{~K}$ and atmospheric pressure. The chains have been unfolded and arranged such that the centers of mass of all the chains are in the central simulation box.

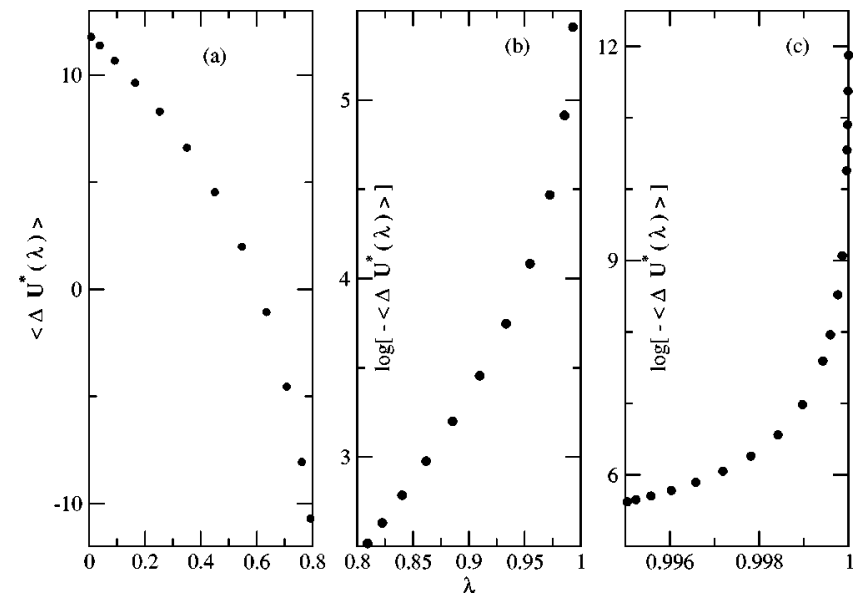

FIG. 4. $\Delta U^{*}(\lambda) \equiv(\beta / N)\left\langle U^{p m}-U^{Y}\right\rangle_{\lambda}$ as a function of the coupling parameter $\lambda$ for $P=400 \mathrm{MPa}$ and $T=435 \mathrm{~K}$.

(b) and (c), we plot the logarithm of the negative of the integrand. For $\lambda=1$, we simulate pure Yukawa particles using Monte Carlo simulation and randomly identify the particles with the polymer atoms to calculate the integrand. The integrand goes smoothly (albeit rapidly) to the limiting value at $\lambda=1$ as shown in panel (c). This shows that even though the integrand is large, our simulations do not suffer from equilibration problems.

In Fig. 5, we show the functions $f_{0}(\Delta U)$ and $f_{1}(\Delta U)$ for $P=400 \mathrm{MPa}$. They overlap for a wide range of energy values. The difference matches with the Widom estimate ${ }^{35}$ of the chemical potential. In the inset of Fig. 5 we show the estimate of $\beta \mu_{\mathrm{ex}}$ for a number of pressures at temperature $435 \mathrm{~K}$.

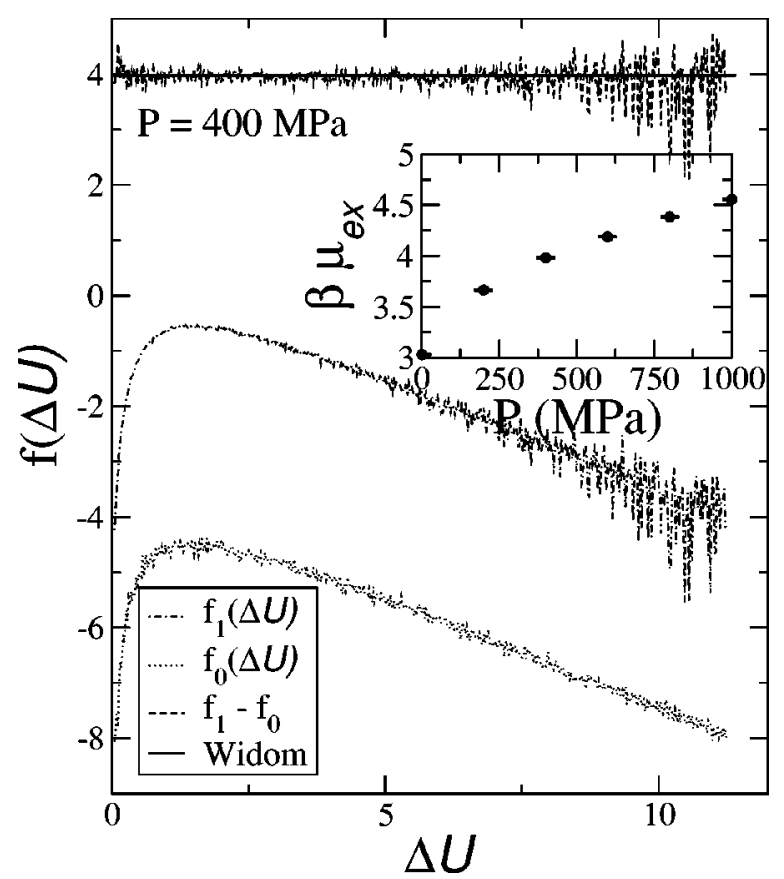

FIG. 5. Estimate of excess chemical potential for reference Yukawa fluid using overlapping distribution function method. Inset: excess chemical potential at $T=435 \mathrm{~K}$ as a function of pressure. See text for the definitions. 


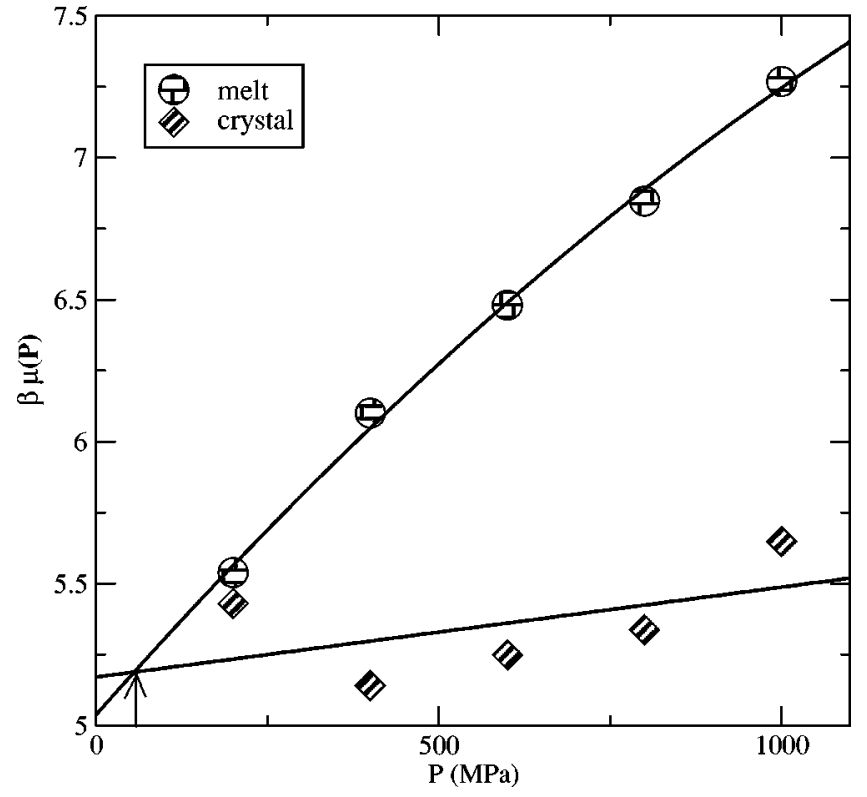

FIG. 6. Configurational part of chemical potential for the melt and the crystal structure as a function of pressure at $435 \mathrm{~K}$. The arrow at $P$ $=55.58 \mathrm{MPa}$ indicates our estimate for the coexistence pressure at this temperature.

In Fig. 6 we plot the configurational part of the chemical potential as a function of pressure at $435 \mathrm{~K}$ for the polymer melt [Eq. (5)] and for the crystal [Eq. (3)]. Also shown are polynomial fits to the data which intersect at $55.58 \mathrm{MPa}$. We have used a least-square linear fit for the crystalline chemical potential data because of the scatter in the individual data points. Thus the coexistence pressure value is insensitive within the error bars to contributions from the individual data points. The largest possible source of error is in the calculation of the melt free energy, where we try to integrate a steep function while coupling to the Yukawa potential. For calculation of the crystalline free energy, determining the correct lattice positions by averaging over the individual particle positions is the main source of error. From our estimates of the statistical errors in the separate steps, the maximum error in the chemical potential to locate the coexistence pressure is of the order of $0.1 k_{B} T$ per atom. From Fig. 6 this translates to about $100 \mathrm{MPa}$ in the coexistence pressure.

From separate simulations of the crystal and the melt at the coexistence point determined from the free energy calculations, we calculate the slope of the coexistence line [Eq. (6) $]$ as $\partial T_{\text {coex }} / \partial P=0.2588(1) \mathrm{K} / \mathrm{MPa}$ at $55.58 \mathrm{MPa}$. This estimate is in excellent agreement with the extrapolated experimental value of $0.23 \mathrm{~K} / \mathrm{MPa}$ from Ref. 38. This gives estimates of the coexistence temperature at other pressures. Simulations at these estimated points provide us with slope informations at all the pressure values considered. We try to fit a smooth cubic polynomial through the state points including the slope informations and get new estimates of the coexistence temperatures. The procedure is repeated until convergence is achieved. ${ }^{39}$

In Fig. 7 we plot the phase diagram obtained from our simulations. The error bars indicate the effect of having an error of $0.1 k_{B} T$ per particle in the free energy calculation.

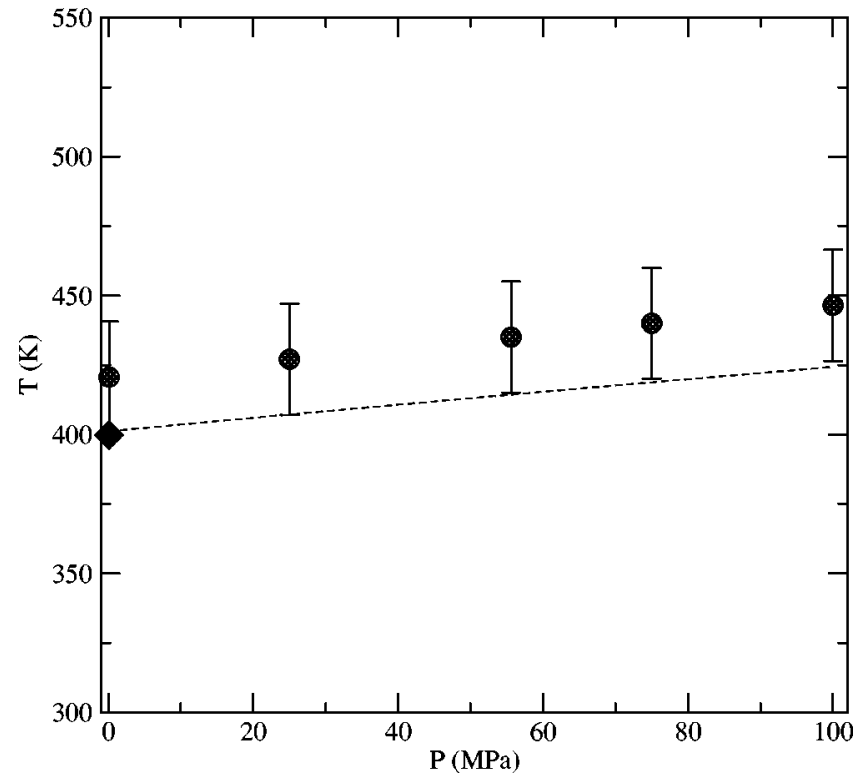

FIG. 7. Melting phase diagram of $\mathrm{C}_{198} \mathrm{H}_{398}$. The circles are from our simulations. The error bars assume $0.1 k_{B} T$ error in chemical potential calculation to locate the coexistence pressure. The diamond is from DSC measurement estimate at ambient pressure of Ungar et al. (Ref. 10) and the dashed line is an interpolation of the experimental phase diagram of Höhne et al. (Ref. 38).

Also shown are the experimental melting temperature at ambient pressure for $\mathrm{C}_{198} \mathrm{H}_{398}$ from Ref. 10 and the extrapolated experimental phase diagram from Ref. 38 .

\section{CONCLUSIONS}

We show that a simple all-atom model of hydrocarbons can predict the experimentally determined crystal structure of long PE oligomers with reasonably good quantitative agreement. The calculated melting phase diagram agrees well with the experimental results. The melting temperature at ambient pressure of $420.6 \pm 20 \mathrm{~K}$ from our simulation corresponds rather well to the DSC measurement estimates of $399.8(3) \mathrm{K}^{10}$ The crystal in our simulations is somewhat more stable than in experiments (we overestimate the melting temperature by almost $20 \mathrm{~K}$ ). The error in the free energy calculation partly accounts for the deviation. Also, because of the small number of molecules in a periodic system, the crystalline structure might be artificially more stable in our simulations.

The melting phase diagram of $n$-alkanes depend on the chain length. One can calculate the phase diagram for different chain lengths and extrapolate to the infinite chain limit. Because of the large costs involved in performing the simulations with much larger systems or much longer chain lengths are not feasible with current computing facilities. Polyethylene has a stable hexagonal phase above $400 \mathrm{MPa}^{40}$ It has been speculated ${ }^{7}$ that nucleation of metastable mobile hexagonal phase and subsequent transformation to less mobile orthorhombic phase might be of importance for the lameller crystal formation. We have not considered the hexagonal phase in our simulation. For calculation of the crystalline free energy, we have used $100 \mathrm{ps}$ for equilibration at the different pressures. While this time is sufficient for relax- 
ing the system to the structure with the same symmetry as the initial configuration, a structural transition would require a much longer time scale. In principle we could explicitly start from the hexagonal structure and repeat the free energy calculation to study its stability in the phase diagram.

Simulations of simple model polymers like bead-spring models and lattice polymers have provided a plethora of insight about polymer melting. However, freezing in a particular crystal structure depends on the specificity of the potential. So, distinguishing between related but different theories is not possible with mean-field-like model systems. We believe that simple potentials which keep all the atoms as in our simulation would be required to resolve such remaining issues.

\section{ACKNOWLEDGMENTS}

This work is part of the research program of the "Stichting voor Fundamenteel Onderzoek der Materie (FOM)," which is financially supported by the "Nederlandse organisatie voor Wetenschappelijk Onderzoek (NWO)." Access to supercomputer TERAS was possible by a grant from Stichting Nationale Computer Faciliteiten (NCF). C.D. acknowledges the financial support provided by the NWO Prioriteits Programma Materialenonderzoek (PPM). We thank Mark Miller and Wenbing $\mathrm{Hu}$ for careful reading of the manuscript.

\section{APPENDIX: THE POTENTIAL MODEL}

The phenomenological potential we used in our simulations has the form,

$$
U=U_{\text {bond }}+U_{\text {angle }}+U_{\text {torsion }}+U_{\text {nonbonded }} \text {. }
$$

The covalent bonds are modeled as harmonic springs, i.e.,

$$
V_{b}=k_{b}\left(b-b_{0}\right)^{2} \text {, }
$$

with the parameters given by ${ }^{25}$

\begin{tabular}{ccc} 
Bond, $b$ & $k_{b}\left(\mathrm{kcal} /\left(\mathrm{mol} \AA^{2}\right)\right)$ & $b_{0}(\AA)$ \\
\hline H-C & 230.0 & 1.04 \\
C-C & 235.5 & 1.53
\end{tabular}

The bond angle energy is assumed harmonic for bond fluctuations, given by

$$
V_{\theta_{i}}=k_{\theta_{i}}\left(\theta_{i}-\theta^{*}\right)^{2}
$$

The stiffness constants and the equilibrium angles used in the simulation are

\begin{tabular}{lcc} 
Angle, $\theta$ & $k_{\theta}\left(\mathrm{kcal} /\left(\mathrm{mol} \mathrm{rad}^{2}\right)\right)$ & $\theta^{*}$ \\
\hline $\mathrm{H}-\mathrm{C}-\mathrm{H}$ & 37.8 & $106^{\circ}$ \\
$\mathrm{H}-\mathrm{C}-\mathrm{C}$ & 44.9 & $110^{\circ}$ \\
$\mathrm{C}-\mathrm{C}-\mathrm{C}$ & 60.0 & $112^{\circ}$
\end{tabular}

The torsion angle is defined by the angle that the normals to the planes defined by three consecutive bonds make with each other. The energy for torsion is fitted as $V\left(\phi_{i}\right)$ $=\Sigma_{n} k_{n}^{T}\left[1+\cos \left(n \phi_{i}\right)\right]$, and the values of $k_{n}^{T}$ used are

\begin{tabular}{ccc} 
Dihedral angle, $\phi$ & $k^{T}(\mathrm{kcal} / \mathrm{mol})$ & $n$ \\
\hline H-C-C-H & 0.165 & 3 \\
H-C-C-C & 0.177 & 3 \\
C-C-C-C & 0.410 & 1 \\
C-C-C-C & 0.096 & 2 \\
C-C-C-C & 0.234 & 3
\end{tabular}

For atoms in different chains or atoms in different parts of the same chain of which the interaction is not taken care by the bending and torsion terms, the potential is given by

$$
v(r)=b e^{-c r}+\frac{a}{r^{6}},
$$

with the parameters given by ${ }^{28}$

\begin{tabular}{lccc} 
Atoms & $b / \mathrm{MJ} \mathrm{mol}^{-1}$ & $c / \AA^{-1}$ & $a / \mathrm{kJ} \mathrm{mol}^{-1} \AA^{6}$ \\
\hline $\mathrm{CC}$ & 259.0 & 3.60 & -2113 \\
$\mathrm{CH}$ & 46.0 & 3.67 & -536 \\
$\mathrm{HH}$ & 11.0 & 3.74 & -135
\end{tabular}

${ }^{1}$ A. Keller and G. Goldbeck-Wood, in Comprehensive Polymer Science, 2nd Supplement, edited by S. L. Aggarval and S. Russo (Elsevier, New York, 1996), pp. 241-305.

${ }^{2}$ J. I. Lauritzen, Jr. and J. D. Hoffman, J. Res. Natl. Bur. Stand., Sect. A 64A, 73 (1960).

${ }^{3}$ D. M. Sadler, Polymer 24, 1401 (1983).

${ }^{4}$ A. Keller et al., J. Mater. Sci. 29, 2579 (1994).

${ }^{5}$ P. D. Olmsted et al., Phys. Rev. Lett. 81, 373 (1998).

${ }^{6}$ M. Imai, K. Kaji, and T. Kanaya, Phys. Rev. Lett. 71, 4162 (1993).

${ }^{7}$ G. Strobl, Eur. Phys. J. E 3, 165 (2000).

${ }^{8}$ J. P. K. Doye and D. Frenkel, J. Chem. Phys. 110, 2692 (1999).

${ }^{9}$ M. Muthukumar, Eur. Phys. J. E 3, 199 (2000).

${ }^{10} \mathrm{G}$. Unger et al., Science 229, 386 (1985).

${ }^{11}$ G. Unger et al., Phys. Rev. Lett. 85, 4397 (2000).

${ }^{12}$ D. Frenkel and B. Smit, Understanding Molecular Simulations, 2nd ed. (Academic, New York, 2002).

${ }^{13}$ D. Frenkel and A. J. C. Ladd, J. Chem. Phys. 81, 3188 (1984).

${ }^{14}$ D. Frenkel, B. M. Mulder, and J. P. McTague, Phys. Rev. Lett. 52, 287 (1984).

${ }^{15}$ S. J. Singer and R. Mumaugh, J. Chem. Phys. 93, 1278 (1990).

${ }^{16}$ A. P. Malanoski and P. A. Monson, J. Chem. Phys. 110, 664 (1999).

${ }^{17}$ E. J. Meijer et al., J. Chem. Phys. 92, 7570 (1990).

${ }^{18}$ B. Kuchta and R. D. Etters, Phys. Rev. B 45, 5072 (1992).

${ }^{19}$ L. A. Baez and P. Clancy, Mol. Phys. 86, 385 (1995).

${ }^{20}$ J. M. Polson and D. Frenkel, J. Chem. Phys. 111, 1501 (1999).

${ }^{21}$ J. M. Polson and D. Frenkel, J. Chem. Phys. 109, 318 (1998).

${ }^{22}$ D. A. Kofke, Mol. Phys. 78, 1331 (1993).

${ }^{23}$ S. Toxvaerd, J. Chem. Phys. 93, 4290 (1990).

${ }^{24}$ J. P. Ryckaert, Mol. Phys. 55, 549 (1985).

${ }^{25}$ D. J. Tobias, K. Tu, and M. L. Klein, J. Chim. Phys. Phys.-Chim. Biol. 94, 1482 (1997).

${ }^{26}$ P. Hobza et al., J. Comput. Chem. 18, 1136 (1997).

${ }^{27}$ R. Martoň́k, W. Paul, and K. Binder, J. Chem. Phys. 106, 8918 (1997).

${ }^{28}$ D. E. Williams, J. Chem. Phys. 47, 4680 (1967).

${ }^{29}$ J. C. Smith and M. Karplus, J. Am. Chem. Soc. 114, 801 (1992).

${ }^{30}$ M. Tuckerman, B. J. Berne, and G. J. Martyna, J. Chem. Phys. 97, 1990 (1992).

${ }^{31}$ G. J. Martyna et al., Mol. Phys. 87, 1117 (1996).

${ }^{32}$ J. Han, R. L. Jaffe, and D. Y. Yoon, Macromolecules 30, 7245 (1997).

${ }^{33}$ C. H. Bennett, J. Comput. Phys. 22, 245 (1976). 
${ }^{34}$ K. S. Shing and K. E. Gubbins, Mol. Phys. 49, 1121 (1983).

${ }^{35}$ B. Widom, J. Chem. Phys. 39, 2802 (1963).

${ }^{36}$ H. Flyvbjerg and H. G. Petersen, J. Chem. Phys. 91, 461 (1989).

${ }^{37}$ G. T. Davis, R. K. Eby, and J. P. Colson, J. Appl. Phys. 41, 4316 (1970).
${ }^{38}$ G. W. H. Höhne and K. Blankenhorn, Thermochim. Acta 238, 351 (1994).

${ }^{39}$ P. G. Bolhuis, M. H. J. Hagen, and D. Frenkel, Phys. Rev. E 50, 4880 (1994).

${ }^{40}$ M. Hikosaka et al., Polymer 33, 2502 (1992). 